\title{
D-10 ENHANCING STRUCTURAL ANALYSIS WITH THE FOURIER TRANSFORM - AN EXAMPLE FROM THE BERKINE BASIN, ALGERIA
}

W. LANSING TAYLOR and FRANCOIS J. GAUTHIER Anadarko Petroleum Corporation, 17001 Northchase Drive, Houston TX 77060, USA

\begin{abstract}
Introduction
The primary challenge of structural geologists in the petroleum industry is to characterize the spatial distribution of folds, faults, and joints in the subsurface and to understand how these features effect the migration and entrapment of hydrocarbons. In most cases, subsurface data are insufficient to accurately characterize rock deformation at the desired scale. As a result, conceptual models are invoked to help translate sparse data sets into structural interpretations. The selection of an appropriate model is often left to intuition or is based on a "common perception" that has not been verified.
\end{abstract}

Fourier analysis has been used extensively in the processing of seismic data. In this context, the technique is used to convert seismic signals recorded in time into the frequency domain, where additional processing and filtering can enhance data quality. We contend that seismic data is not the only data set that benefits from analysis in the frequency domain. Just as the seismic response recorded at a geophone varies through time as energy is reflected back from the subsurface, the depth to the top of a given formation varies through space as folds and faults are crossed. Fourier analysis can be used to extract information on the amplitude, spacing, and orientation of these folds and faults, assisting with the interpretation and integration of sparse data sets into mechanically consistent and geologically meaningful maps.

We have used Fourier analysis as one component of our structural synthesis in the Berkine Basin, Algeria. The analysis has provided an objective means of filtering our data and quantifying the geometric attributes of subsurface structures. Along the HBNS - El Borma producing trend, we have been able to quantitatively characterize the spatial distribution of three major fault and fold sets that together control the location and size of many of the fields in this producing region. We have also developed specific scaling relationships between large-scale and small-scale features that help us to predict deformation in regions of poor data coverage where only large features are discernible.

\section{Use of the Fourier Transform in Structural Analysis}

The basic concept underlying Fourier analysis is that adding together simple trigonometric functions can reproduce any complex surface, such as a geologic horizon (figure 1). These trigonometric functions can be completely described by four parameters: (1) amplitude, (2) wavelength, (3) orientation, and (4) phase. The first three of these parameters can be easily displayed on a polar plot where the distance from the origin represents the wavelength, the radial angle represents the orientation, and the size, color, or label associated with each data 


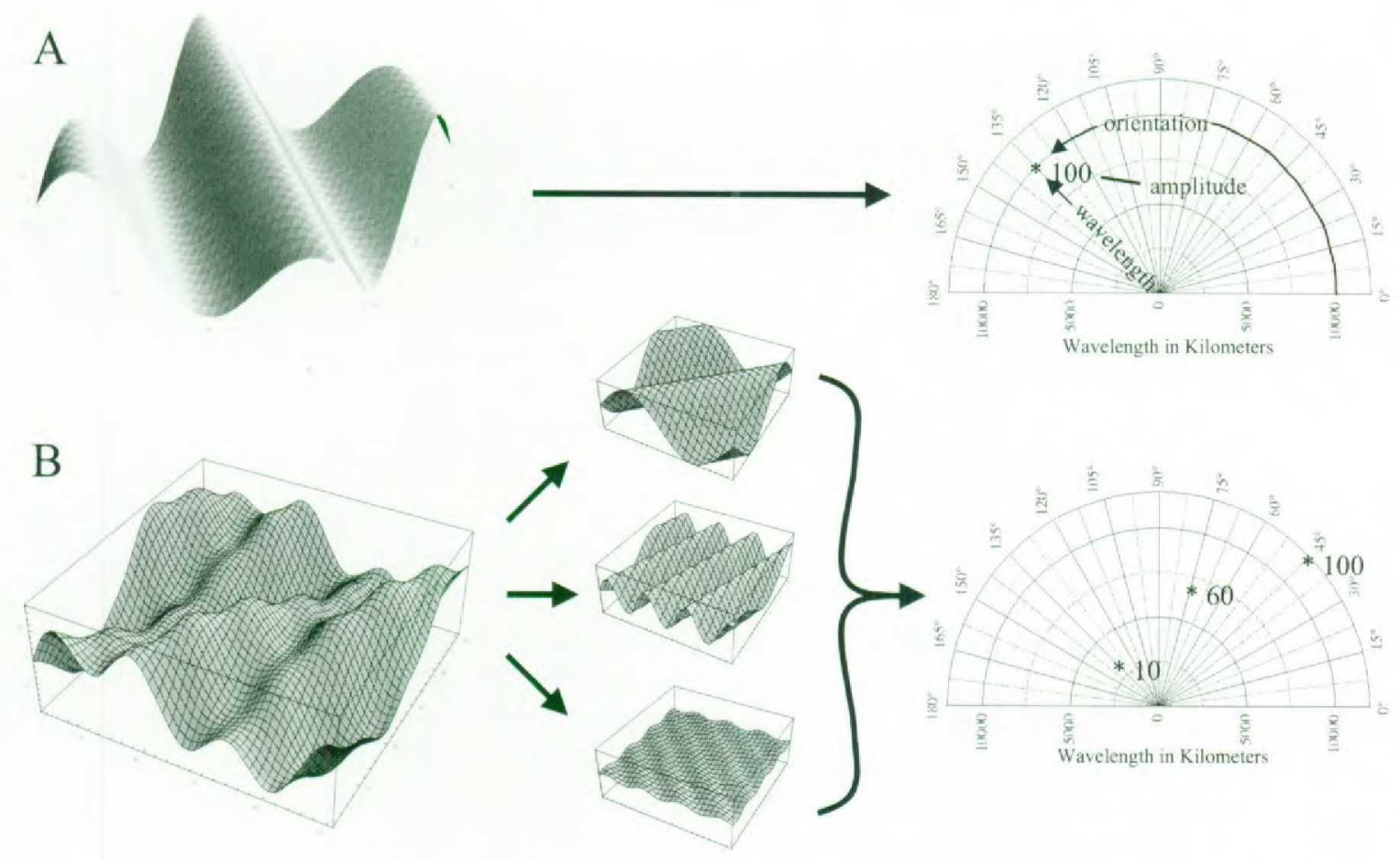

1. A) The amplitude, wavelength, orientation, and phase uniquely describe a trigonometric surface. B) Any complex surface can be represented as the sum of trigonometric functions.

point corresponds to the amplitude. The Fourier transform is a mathematical operation that deconvolves a complex surface into its constitutive components.

Deformation within the Earth is not randomly distributed. There are physical processes acting on and within a rock mass that impart a consistent spacing and orientation to folds, faults, and joints. For example, the wavelength of a fold can be related to the mechanical properties of the folded layers (figure 2). Similarly, faults and joints are both associated with a perturbed stress field that inhibits further deformation in the adjacent rock mass. The size of the perturbation scales with the dimensions of the fault or joint surface, so in a well

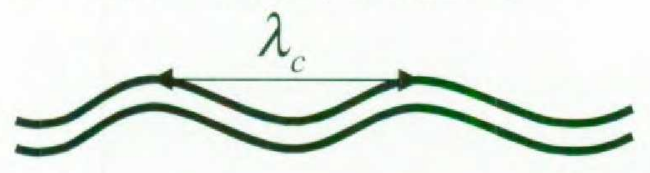

$$
\lambda_{c}=2 \pi\left(\frac{E h^{3}}{12\left(1-v^{2}\right)\left(\rho_{m}-\rho_{w}\right) g}\right)^{1 / 4}
$$

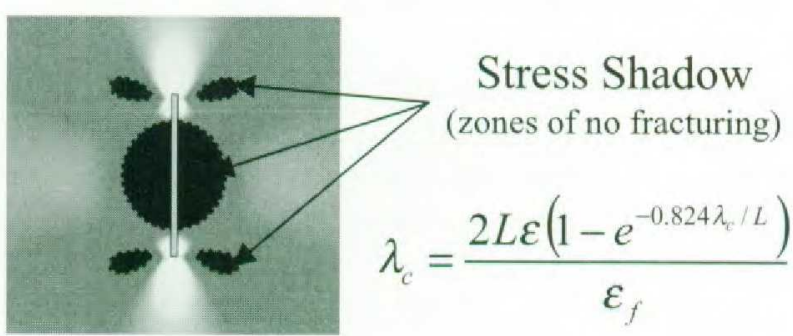

2. The mechanical behavior of rock naturally produces periodicity in the spatial distribution of folding, faulting, and jointing. In these examples, $\lambda_{c}$ is the wavelength of the fold or the spacing between adjacent faults or joints. $\lambda_{c}$ depends on rock properties such as density, $\rho$, Young 's Modulus, E, and Poisson's Ratio, $v$. It also depends on the geometry of the rock layers and the loading conditions. Fourier analysis aids in the quantitative characterization of structural periodicity. 


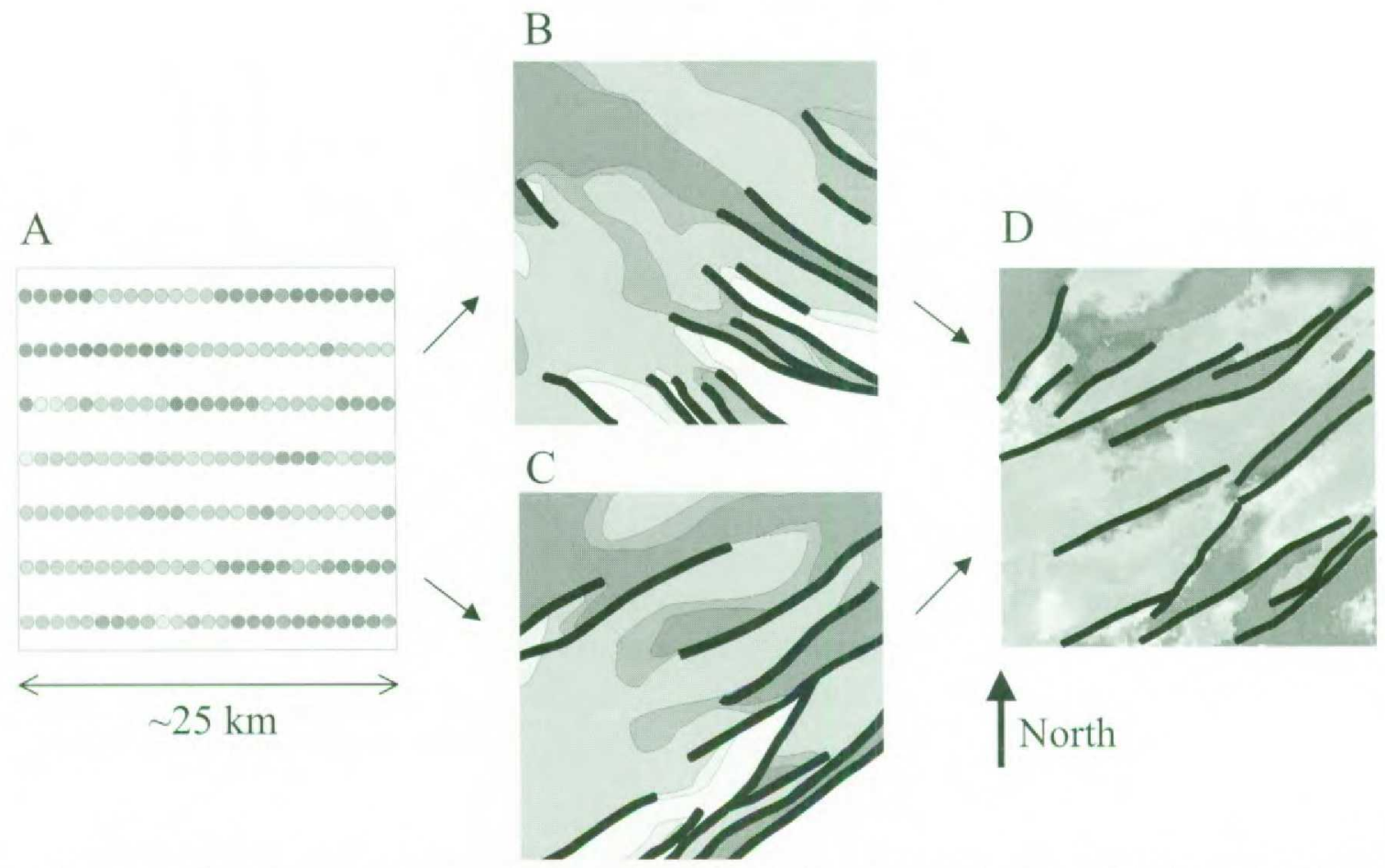

3. An example of how Fourier analysis may improve fault interpretation on 2D seismic data. A) Decimated data from a 3D survey. Each circle represents a data point, so there are significant portions of the subsurface with no data. The gray scale corresponds to depth below sea level. B) The "most plausible" interpretation based on visual inspection of the data. C) An interpretation conditioned with Fourier analysis. D) The "known" answer from the 3D survey.

developed (saturated) system, the spacing of faults and joints is typically proportional to their length or height. We suggest that much natural deformation is periodically distributed, meaning that the distance between adjacent features with similar dimensions is about the same from one feature to the next.

The transformation of a faulted and folded geologic horizon into the frequency domain produces a new data set that highlights periodic trends in the original data. For a typical industry data set consisting of 2D seismic lines and a few wells, it is often possible to create several alternate structural interpretations, all of which have consistent fault and fold orientations and that satisfy basic scaling relationships, such as the relationship between fault length and fault offset. We propose that the periodicity of deformation can be used as an additional interpretation constraint, and have identified Fourier analysis as the optimum tool for objectively extracting periodic trends from spatially incomplete data.

\section{Applications of Fourier Analysis, Berkine Basin, Algeria}

To test the utility of Fourier analysis in constraining fault interpretations, we created a synthetic $2 \mathrm{D}$ data set by decimating a faulted horizon mapped from a 3D survey (figure 3). We then made the most plausible interpretation of the decimated data set based on a visual 

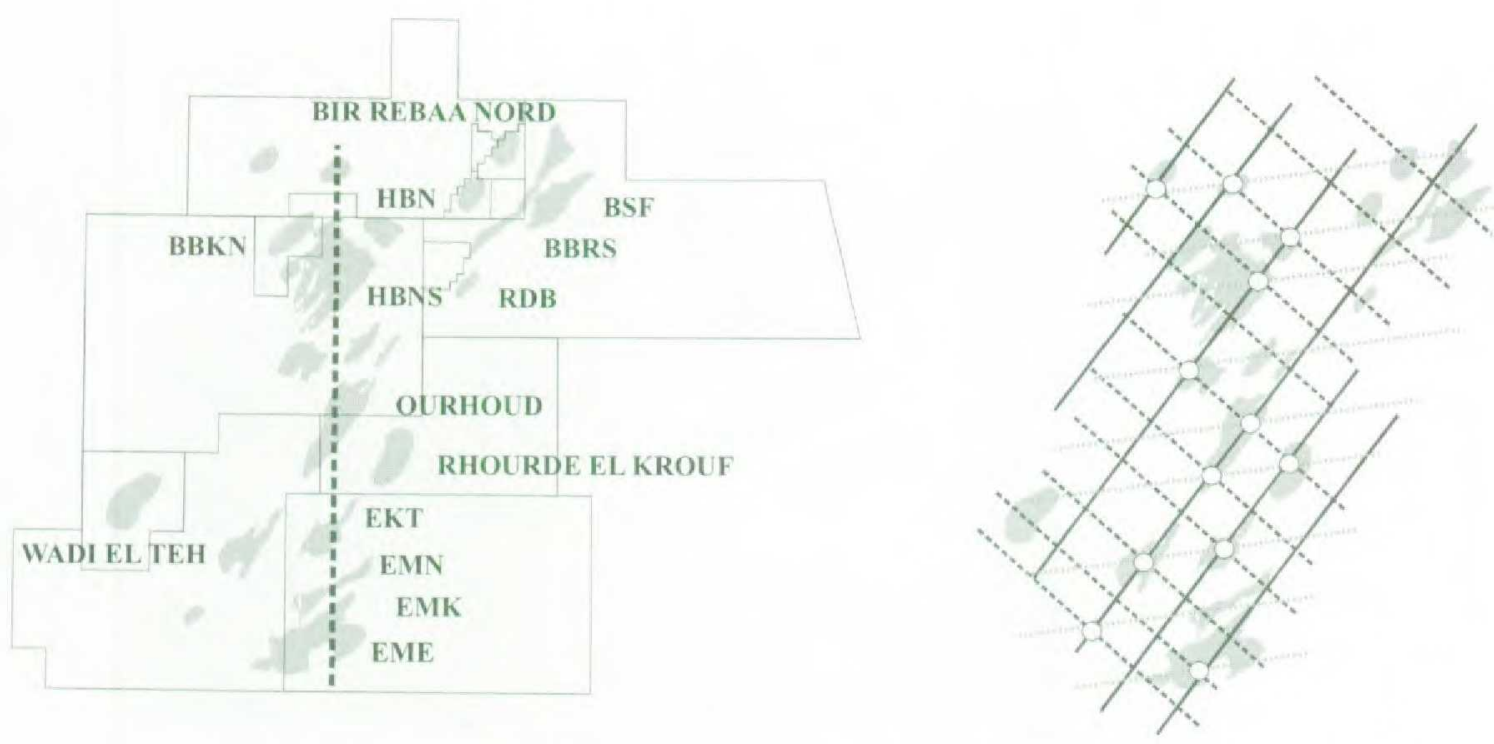

4. The distribution of fields in a portion of the HBNS - El Borma producing trend. Despite the dominant NE-SW striking fault system, the producing trend from EME to HBN appears to be oriented $N-S$. Each major field occurs where three separate fault or fold trends constructively interact to create a structural closure.

correlation from one line to the next. A prominent NW to SE trending structural low cuts across five of the synthetic 2D lines, suggesting that the major fault trends are oriented NW- SE. Our original interpretation reflects this trend. We also ran a Fourier analysis on the 175 decimated data points and found that the dominant periodic trend in the data strikes NE-SW. A second interpretation was constructed, again by visually correlating from one line to the next, but now preserving this alternate orientation of faulting even though the trend appears to cross cut the visually prominent NW-SE trend. Comparing the two interpretations to the known horizon from the 3D survey, we found that the Fourier analysis successfully extracted the appropriate fault orientation and that the conditioned interpretation is a more accurate representation of the actual faulted horizon.

Having tested the approach, we preformed a Fourier analysis on all available subsurface horizon data for the top of the TAGI sandstone, the major hydrocarbon reservoir along the HBNS - El Borma producing trend. The analysis affirmed that three fault and fold systems previously identified from 3D seismic data are regionally pervasive and extend into areas where they are not directly imaged on poorer quality $2 \mathrm{D}$ data. Along the main producing trend, the dominant fault set strikes NE-SW but the alignment of fields is roughly N-S (figure 4). By using the Fourier analysis to quantify the periodicity of these structural trends and project them into areas of poor data coverage, we have identified that more than $75 \%$ of the producing fields occur where the three fault and fold trends intersect to create structural closures. It is the alignment of the intersections between these trends that is oriented N-S and that defines the distribution of producing fields in the basin.

Our analysis has provided a comprehensive data set of fault length and fault offset in the Berkine basin. There is a developing body of literature arguing that fault populations have a fractal distribution, with fault density linearly related to fault dimensions on a log-log plot (for example, see Heffer \& Beven, 1990, or Childs et al., 1990). In contrast, our Fourier analysis indicates that fault lengths in the Berkine basin cluster around $40 \mathrm{~km}, 8 \mathrm{~km}$, and $1.5 \mathrm{~km}$, with vertical offsets around $140 \mathrm{~m}, 40 \mathrm{~m}$, and $15 \mathrm{~m}$ respectively. In sedimentary basins, fault length, height, and offset are strongly influenced by the lithology of competent formations, and the thickness of individual beds (for example, see Scholz \& Contreras, 1998, or Steen \& Andresen, 1999). Because fault dimensions are related to mechanical heterogeneity within the basin, clustering in fault populations is expected. In the Berkine basin, our Fourier analysis indicates a consistent geometric scaling between the mean values of adjacent fault population clusters that covers at least five orders of magnitude. Rather than a linear relationship, this appears as a sinusoidal relationship on a log-log plot with a consistent wavelength but decreasing 
amplitude at larger fault dimensions. The regular periodicity of this sinusoid provides an alternate, mechanically plausible, statistical model for predicting sub-seismic fạult distributions.

\section{References}

Childs, C., Walsh, J. J., and Watterson, J. 1990. A method for estimation of the density of fault displacements below the limits of seismic resolution in reservoir formations. North Sea Oil and Gas Reservoirs; II. Proceedings of the North Sea Oil and Gas Reservoirs Conference. ed. Graham \& Trotman. p. 309-318.

Heffer, K. J., and Bevan, T. G. 1990. Scaling relationships in natural fractures - data, theory and applications. SPE Reprint 20981, 1-12.

Scholz, C. H., and Contreras, J. C. 1998. Mechanics of continental rift architecture. Geology; v. 26; no. 11 ; p. 967-970.

Steen, O., Andresen, A. 1999. Effects of lithology on geometry and scaling of small faults in Triassic sandstones, East Greenland. Journal of 